\title{
Phycobiliprotein genes of the marine photosynthetic prokaryote Prochlorococcus: evidence for rapid evolution of genetic heterogeneity
}

\author{
Claire S. Ting, ${ }^{1}$ Gabrielle Rocap ${ }^{1,2}$ Jonathan King ${ }^{1}$ \\ and Sallie W. Chisholm ${ }^{1,2}$
}

Department of Biology 1 and Department of Civil and Environmental Engineering ${ }^{2}$

Massachusetts Institute of Technology, Cambridge, MA 02139, USA
Author for correspondence: Sallie Chisholm. Tel: +1 617253 1771. Fax: +1 6172587009. e-mail: chisholm@mit.edu

Prochlorococcus is a major photosynthetic prokaryote in nutrient-limited, open ocean environments and an important participant in the global carbon cycle. This phototroph is distinct from other members of the cyanobacterial lineage to which it belongs because it utilizes a chlorophyll $a_{2} / b_{(2)}$ light-harvesting complex as its major antenna, instead of phycobilisomes. Recently, genes encoding the phycobiliprotein phycoerythrin were identified in several Prochlorococcus isolates, thus making it the only extant photosynthetic prokaryote to possess a chlorophyll alb antenna as well as phycobiliprotein genes. In order to understand the evolution of phycobiliproteins in this genus, the authors have sequenced the phycoerythrin genes of two isolates that are the most deeply branching in the Prochlorococcus lineage and share the highest degree of $16 \mathrm{~S}$ rDNA sequence similarity to phycobilisome-containing marine Synechococcus. Sequence analyses suggest that within the Prochlorococcus lineage, the selective forces shaping the evolution of the phycoerythrin gene set have not been uniform. Although strains that are most closely related to marine Synechococcus possess genes (cpeB, cpeA) encoding both subunits of phycoerythrin, a more recently evolved strain is shown to lack cpe $A$ and to possess a degenerate form of cpeB. Differences in phycoerythrin gene sequences between Prochlorococcus and Synechococcus appear to be consistent with a model of elevated mutation rates rather than relaxed selection. This suggests that although phycoerythrin is not a major constituent of the light-harvesting apparatus in Prochlorococcus, as it is in Synechococcus, the cpe $B$ and cpe $A$ genes are still under selection, albeit a different type of selection than in Synechococcus. The evolution of the Prochlorococcus lightharvesting antenna complex provides an important system for understanding the origins and scope of phylogenetic diversity in ocean ecosystems.

Keywords: cyanobacteria, light-harvesting complex, phycoerythrin, phylogeny, relative rates of evolution

\section{INTRODUCTION}

The oceans are responsible for nearly half of the earth's photosynthetically fixed carbon. In tropical and subtropical marine environments, the unicellular pro-

\section{Abbreviations: Chl, chlorophyll; PE, phycoerythrin.}

The GenBank accession numbers for the sequences reported in this paper are AF304132 (MIT9303 cpeB), AF304135 (MIT9303 cpeA), AF304133 (MIT9313 cpeB) and AF304134 (MIT9313 cpeA). karyotes Prochlorococcus and Synechococcus are dominant members of the photosynthetic biomass (Partensky et al., 1999). Phylogenetic studies based on analyses of $16 \mathrm{~S}$ rRNA and other ( $p s b B$, psbA, petB/D operon, rpoC1) genes indicate that Prochlorococcus is most closely related to phycobilisome-containing, marine Synechococcus species (marine cluster-A) (Palenik \& Haselkorn, 1992; Urbach et al., 1992, 1998; Hess et al., 1995). Branching orders between Prochlorococcus and marine Synechococcus are not well resolved and suggest 
a rapid and almost simultaneous diversification of both groups from a common ancestor (Urbach et al., 1998).

Although Prochlorococcus and Synechococcus share close phylogenetic ties and several ecological and physiological characteristics, a fundamental difference exists between these genera in their photosynthetic light-harvesting apparatus. A major antenna of the latter consists of phycobiliproteins that are covalently bound to linear tetrapyrrole prosthetic groups and are organized into phycobilisomes (Bryant, 1991). The phycobilisomes of marine cluster-A Synechococcus are phycoerythrin (PE)-rich, and two types of PE (I, II) have been identified that differ in their number of associated bilins (Ong \& Glazer, 1991; Wilbanks et al., 1991). In contrast, the major antenna complex of Prochlorococcus is based on the pigments divinyl chlorophyll $a\left(\mathrm{Chl} a_{2}\right)$ and divinyl and/or monovinyl chlorophyll $b$ (Chl $b_{2}$, Chl b) (Chisholm et al., 1992; Goericke \& Repeta, 1992; LaRoche et al., 1996; Partensky et al., 1997). Its light-harvesting complex polypeptides ( $\mathrm{Pcb})$ are members of the Chl a-binding core complex family of antenna proteins, which includes CP43 and CP47 (LaRoche et al., 1996; Partensky et al., 1997).

Recently, genes encoding the $\alpha(c p e A)$ and $\beta$ (cpeB) subunits of PE were identified in Prochlorococcus sp. strain SS120 (CCMP1375) (Hess et al., 1996). In SS120, both $c p e B$ and $c p e A$ are found in a gene cluster containing genes that are homologous to those encoding other phycobiliproteins (MpeC, CpeZ) and bilin biosynthesis proteins (Hess et al., 1999). Although low levels of $c p e B$ and $c p e A$ expression have been detected in SS120 (Hess et al., 1996, 1999), the functional significance of PE in SS120 is unknown. PE genes have since been found in Prochlorococcus isolates PAC1 and PAC2 (Penno et al., 2000), and we have also identified среB in the genome of Prochlorococcus MED4. This latter isolate is capable of growing at higher ( $>300 \mu \mathrm{mol}$ photons $\mathrm{m}^{-2} \mathrm{~s}^{-1}$ ) irradiance levels than low-light-adapted Prochlorococcus isolates such as SS120, and is a member of the high-light-adapted clade (Urbach et al., 1998; Moore et al., 1998; Rocap, 2000).

We have in our collection two low-light-adapted Prochlorococcus isolates, MIT9303 and MIT9313, which are the most deeply branching in the Procblorococcus lineage (Moore et al., 1998; Rocap, 2000). In this unique phylogenetic position, their $16 \mathrm{~S}$ rDNA sequences differ by approximately $2-3 \%$ from other isolates of both Prochlorococcus sp. and marine Synechococcus sp. In order to obtain a more complete understanding of the evolution of phycobiliproteins in Prochlorococcus, we sequenced the $c p e B$ and $c p e A$ genes of both MIT9303 and MIT9313. Our analyses suggest that within the Prochlorococcus lineage the selective forces shaping the evolution of the PE gene set have not been uniform. Furthermore, the PE gene sequence heterogeneity we document between Prochlorococcus and Synechococcus is consistent with a model of elevated mutation rates, rather than relaxed selection. Elucidating the rates and pathways of genetic change in this important phototroph may help in assessing its response to rapid environmental change.

\section{METHODS}

Culture conditions. Prochlorococcus isolates MIT9303 and MIT9313, and marine Synechococcus sp. strain WH8103, were grown in batch cultures in Sargasso Sea water supplemented with 'Pro2' medium nutrients and trace metals (Moore \& Chisholm, 1999). Cultures were maintained at $21 \pm 1{ }^{\circ} \mathrm{C}$. Illumination was supplied during a $14 \mathrm{~h}$ light: $10 \mathrm{~h}$ dark cycle by 'cool white' fluorescent lights at $10 \mu \mathrm{mol}$ photons $\mathrm{m}^{-2} \mathrm{~s}^{-1}$.

Genomic DNA isolation. Genomic DNA was prepared from Prochlorococcus according to standard methods (Ausubel et al., 1995) that were optimized for this bacterium (Rocap, 2000). Briefly, cells were lysed in the presence of lysozyme, and DNA was extracted with phenol/chloroform/isoamyl alcohol (25:24:1, by vol.), precipitated with 2-propanol, and resolubilized in TE buffer ( $\mathrm{pH} 7 \cdot 6$ ).

PCR amplification, cloning and sequencing. Genes encoding the $\beta$ - $(c p e B)$ and $\alpha-(c p e A)$ subunits of PE were amplified from Prochlorococcus genomic DNA by PCR. The primers, cpeB1.2 (ATGCTTGATGCATTCTCAAG) and cpeA 2.2 (AAGGCATTAATAAGGTAATC), were designed as exact matches to the SS120 cpeB 5'-end and cpeA $3^{\prime}$-end (Hess et al., 1996). PCR samples contained 1-3 $\mathrm{g}$ genomic DNA, $0.5 \mu \mathrm{M}$ of each primer, $250 \mu \mathrm{M}$ each dNTP, $0-3.5 \mathrm{mM} \mathrm{MgCl}_{2}$, cloned $P f u$ buffer $(1 \times)$ (Stratagene), and 2.5 units $P f u$ DNA polymerase (Stratagene). Cycle parameters were $94^{\circ} \mathrm{C}$ for $1 \mathrm{~min}, 50{ }^{\circ} \mathrm{C}$ for $1 \mathrm{~min}$ and $72^{\circ} \mathrm{C}$ for $2 \mathrm{~min}$. Samples were processed for 30 or 40 cycles prior to a final extension at $72^{\circ} \mathrm{C}$ for $10 \mathrm{~min}$.

PCR products were separated on $1.5 \%$ agarose gels and visualized by UV fluorescence after staining with ethidium bromide. Selected PCR products of $\sim 1000 \mathrm{bp}$ were purified from agarose gels using the QIAEXII Kit (QIAGEN), and then ligated to the pCR-Script Amp SK(+) Cloning Vector (PCRScript Amp Cloning Kit, Stratagene). Vectors containing this insert were used to transform Escherichia coli (Epicurian Coli XL 10-Gold Kan ultracompetent cells, Stratagene). Sizeselected clones were sequenced and final $c p e B$ and $c p e A$ sequences were obtained from three clones for MIT9303 and two clones for MIT9313. Plasmids were sequenced using M13 forward and reverse primers in our laboratory using SequiTherm LongRead Cycle Sequencing Kits on LI-COR model 4000L sequencers or by the MITCCR HHMI Biopolymers Laboratory.

Phylogenetic analysis. Sequences used in comparisons were obtained from GenBank and from the complete MED4 genome (http://spider.jgi-psf.org/JGI_microbial/html/). All analyses utilized $c p e B$ sequences minus the first 20 nucleotides ( $5^{\prime}$ primer region) and cpeA minus the last 24 nucleotides ( $3^{\prime}$ primer region). Data and alignments for $16 \mathrm{~S} \mathrm{rDNA}$ analyses were downloaded directly from the Ribosomal Database Project (RDP) (Maidak et al., 2000).

PE protein sequences were aligned using the CLUSTAL $\mathrm{W}$ program with the вLOsum matrix (Thompson et al., 1994). Gaps were included to optimize the alignments. Only unambiguously aligned positions were used in the phylogenetic analyses (146 amino acids for $\alpha$-PE, 159 amino acids for $\beta$-PE). The percentage identities summarized in Table 1 were calculated from uncorrected distances. Total G $+\mathrm{C}$ base content ratios were calculated using GeneMark (Borodovsky 
\& McIninch, 1993). MEgA version 2.0 (Kumar et al., 2001) was used to calculate the number of synonymous and nonsynonymous substitutions using both the original and modified Nei-Gojobori method $(R=1.28$ for $c p e B, R=1.35$ for $c p e A$ ) (Nei \& Gojobori, 1986; Ina, 1995; Nei \& Kumar, 2000; Kumar et al., 2001). The numbers of synonymous and nonsynonymous differences were compared using $p$-distances, where the number of synonymous differences was normalized to the number of synonymous sites, and the number of nonsynonymous differences was normalized to the number of nonsynonymous sites. Conclusions drawn from the original and modified Nei-Gojobori analyses were the same, and only the results of the latter analyses have been reported. Nonparametric relative rate tests were conducted in MEGA version 2.0 (Kumar et al., 2001) using Tajima's general method (Tajima, 1993), with one degree of freedom and a significance level of $5 \%$.

Protein phylogenetic analyses were done with PUZzLE 4.0 (Strimmer \& von Haeseler, 1996) for maximum-likelihood, PHYLIP 3.5c (Felsenstein, 1989) for distance, and PHYLIP 3.5c (Felsenstein, 1989) for parsimony algorithms. Distances were calculated in PROTDIST using the Dayhoff-PAM substitution matrix and trees were inferred using the Fitch-Margoliash weighted least-squares criterion in FITCH. Distance and parsimony bootstrap analyses were performed with 1000 pseudosampled datasets generated by SEQBOOT. In the protein maximum-likelihood analyses, the JTT substitution model was used and the puzzling step was repeated 1000 times.

PAUP* version 4.0 beta 2 a (Swofford, 1999) was used for all nucleotide analyses. Percentage identities reported in Table 6 were calculated from uncorrected distances. Distance trees were inferred using minimum evolution as the objective criterion and paralinear (logdet) distances, which are relatively insensitive to differences in $\mathrm{G}+\mathrm{C}$ content (Lake, 1994). Nucleotide maximum-likelihood analyses were done via the HKY85 model of nucleotide substitution with rate heterogeneity and empirical nucleotide frequencies. Bootstrap analyses (100 resamplings for maximum-likelihood, 1000 for distance and maximum-parsimony) were performed with heuristic searches utilizing random addition and tree-bisection reconnection branch-swapping methods. Phylogenetic trees were displayed using TREEVIEW (Page, 1996).

Accession numbers. Sequences reported in this paper were deposited in GenBank under the following accession numbers: AF304132 (MIT9303 среB), AF304135 (MIT9303 сpeA), AF304133 (MIT9313 cpeB), AF304134 (MIT9313 cpeA). Database accession numbers of the PE sequences reported for other organisms, including Prochlorococcus (Pro) and Synechococcus (Syn), are as follows: Pro SS120 Z68890; Pro PAC1 AJ272069; Pro PAC2 AJ237612, AJ272030; Syn WH7803 X72961; Syn WH8103 M91809; Syn WH8020 M95288; Synechocystis PCC6701 M33812; Calothrix PCC7601 X04592; Pseudanabaena PCC7409 X63073, S96581; Porphyra purpurea U38804; Aglaothamnion neglectum Z11907; Cryptomonas sp. AFMDB; Chroomonas sp. S21775. Accession numbers for $16 \mathrm{~S}$ rDNA sequences are as follows: Pro MIT9303 AF053397; Pro MIT9313 AF053399; Pro NATL2A AF001467; Pro PAC1 AF001471; Pro MED4 AF001466; Pro SS120 X63140; Syn WH8101 AF001480; Syn WH8103 AF001479; Syn WH7805 AF001478; Syn WH7803 AF081834; Syn PCC6307 AF001477; Syn PCC7001 AB015058; Syn PCC6301 X03538; Syn PCC7942 D88288; Synechocystis PCC6803 D64000; Microcystis holsatica U40336; Microcystis elabens U40335; Arthrospira PCC8005 X70769; Calothrix D253 X99213; Pseudanabaena biceps PCC7367 AF091108; Plectonema F3 AF091110; Nostoc punctiforme PCC73102
AF027655; Pleurocapsa PCC7516 X78681; Cyanidium caldarium chloroplast X52985; Antithamnion chloroplast X54299; Chondrus crispus chloroplast Z29521; Palmaria palmata Z18289; Cryptomonas phi chloroplast S73904; Cyanophora paradoxa cyanelle U30821.

\section{RESULTS}

\section{Amplification, cloning and sequencing of MIT9303 and MIT9313 PE genes}

Using primers that were exact matches to the SS120 cpeB (5'-end) and cpeA ( $3^{\prime}$-end) genes, and Synechococcus sp. strain WH8103 as the control, we optimized the PCR conditions in the presence of sequence mismatches between the primers and the target $c p e B$ and $c p e A$ genes. Although the PE genes of Synechococcus sp. strain WH8103 possessed three mismatches to the $c p e B$ primer $\left(5^{\prime}\right.$ - end) and six mismatches to the cpeA primer ( $3^{\prime}$-end), we amplified a single DNA fragment of the approximate size expected for $c p e B$ and $c p e A(\sim 1000 \mathrm{bp}$ ) (Fig. 1, lane 2). Using the same PCR conditions, we then sought to amplify the genes encoding the $\alpha$ - and $\beta$-subunits of PE from the genomic DNA of MIT9303 and MIT9313. Separation of the MIT9303 PCR products on an agarose gel revealed a single major DNA fragment of $\sim 1000 \mathrm{bp}$ (Fig. 1, lane 3). Although this was also the case for MIT9313, additional minor DNA fragments were observed for this isolate (Fig. 1, lane 4). Subsequent cloning and sequencing of the $\sim 1000 \mathrm{bp}$ DNA fragments from the two isolates identified them as putative $c p e A$ and $c p e B$ genes. Using these gene and derived amino acid sequences, as well as those from SS120 (Hess et al., 1996), we performed a BLAST search of the MED4 genome and were able to identify the MED4 $c p e B$ gene. However, we found that MED4 lacks a cpeA homologue.

\section{Nucleotide sequence comparisons}

The cpeB gene sequences of MIT9303 and MIT9313 exhibited $97 \%$ identity and were more similar to each other than they were to other Prochlorococcus or marine

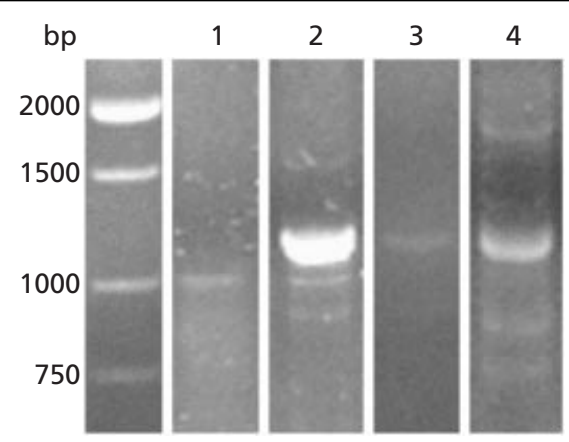

Fig. 1. $P C R$ amplification of Prochlorococcus and marine Synechococcus DNA using a set of exact match primers to the $5^{\prime}$-end of SS120 cpeB and to the $3^{\prime}$-end of SS120 cpeA. The first lane shows a DNA ladder. Lane 1, SS120 (control). Note the single PCR product of $\sim 1000 \mathrm{bp}$. Lane 2, Synechococcus sp. strain WH8103. Lane 3, MIT9303. Lane 4, MIT9313. 
Table 1. Identity matrix for $c p e B$ (bold type) and $\beta$-PE (above the diagonal) and cpe $A$ (bold type) and $\alpha$-PE (below the diagonal) of Prochlorococcus, and other cyanophytes and rhodophytes

\begin{tabular}{|c|c|c|c|c|c|c|c|c|c|c|c|c|c|c|c|c|}
\hline & & 1 & 2 & 3 & 4 & 5 & 6 & 7 & 8 & 9 & 10 & 11 & 12 & 13 & 14 & 15 \\
\hline \multirow[t]{2}{*}{1} & Prochlorococcus & & 97 & 65 & 65 & 64 & 38 & 67 & 60 & 64 & 61 & 43 & 65 & 61 & 51 & 45 \\
\hline & MIT9303 & & 99 & 77 & 78 & 78 & 37 & 66 & 63 & 64 & 61 & 63 & 60 & 55 & 62 & 59 \\
\hline \multirow[t]{2}{*}{2} & Prochlorococcus & 97 & & 65 & 65 & 64 & 38 & 67 & 60 & 64 & 61 & 42 & 52 & 61 & 52 & 46 \\
\hline & MIT9313 & 99 & & 77 & 78 & 78 & 37 & 66 & 64 & 64 & 61 & 62 & 59 & 55 & 62 & 60 \\
\hline \multirow[t]{2}{*}{3} & Prochlorococcus & 66 & 67 & & 77 & 78 & 40 & 46 & 46 & 59 & 46 & 49 & 39 & 44 & 62 & 65 \\
\hline & SS120 & 64 & 64 & & 86 & 86 & 40 & 62 & 61 & 61 & 58 & 58 & 51 & 52 & 61 & 55 \\
\hline \multirow[t]{2}{*}{4} & Prochlorococcus & 62 & 62 & 70 & & 89 & 42 & 49 & 47 & 49 & 48 & 46 & 41 & 44 & 52 & 52 \\
\hline & PAC1 & 63 & 63 & 75 & & 98 & 41 & 65 & 63 & 65 & 60 & 60 & 56 & 54 & 64 & 58 \\
\hline \multirow[t]{2}{*}{5} & Prochlorococcus & 61 & 62 & 70 & 94 & & 42 & 47 & 45 & 47 & 47 & 47 & 41 & 31 & 52 & 54 \\
\hline & PAC2 & 63 & 63 & 75 & 97 & & 41 & 66 & 63 & 66 & 60 & 60 & 56 & 56 & 62 & 59 \\
\hline \multirow[t]{2}{*}{6} & Prochlorococcus & - & - & - & - & - & & 23 & 16 & 23 & 23 & 30 & 36 & 33 & 37 & 35 \\
\hline & MED4 & - & - & - & - & - & & 36 & 32 & 35 & 32 & 34 & 32 & 27 & 32 & 34 \\
\hline \multirow[t]{2}{*}{7} & Synechococcus & 65 & 65 & 59 & 52 & 58 & - & & 69 & 79 & 68 & 63 & 61 & 63 & 64 & 50 \\
\hline & WH7803 (PEI) & 61 & 61 & 57 & 59 & 59 & - & & 69 & 88 & 67 & 74 & 70 & 68 & 73 & 71 \\
\hline \multirow[t]{2}{*}{8} & Synechococcus & 58 & 57 & 13 & 40 & 40 & - & 66 & & 55 & 79 & 63 & 61 & 63 & 64 & 58 \\
\hline & WH8103 (PEII) & 52 & 52 & 48 & 53 & 53 & - & 57 & & 69 & 91 & 65 & 60 & 57 & 69 & 66 \\
\hline \multirow[t]{2}{*}{9} & Synechococcus & 69 & 68 & 61 & 53 & 53 & - & 79 & 63 & & 67 & 66 & 65 & 54 & 66 & 63 \\
\hline & WH8020 (PEI) & 61 & 61 & 57 & 60 & 60 & - & 92 & 57 & & 68 & 74 & 72 & 62 & 72 & 70 \\
\hline \multirow[t]{2}{*}{10} & Synechococcus & 60 & 61 & 42 & 43 & 43 & - & 61 & 83 & 63 & & 48 & 61 & 54 & 62 & 58 \\
\hline & WH8020 (PEII) & 49 & 49 & 49 & 52 & 53 & - & 54 & 91 & 53 & & 66 & 60 & 54 & 68 & 67 \\
\hline \multirow[t]{2}{*}{11} & Synechocystis & 63 & 64 & 61 & 57 & 56 & - & 66 & 59 & 63 & 63 & & 73 & 69 & 68 & 54 \\
\hline & PCC6701 & 59 & 59 & 57 & 61 & 60 & - & 72 & 64 & 70 & 64 & & 87 & 70 & 66 & 66 \\
\hline \multirow[t]{2}{*}{12} & Calothrix & 64 & 63 & 60 & 61 & 53 & - & 65 & 59 & 64 & 62 & 80 & & 74 & 68 & 55 \\
\hline & PCC7601 & 61 & 61 & 58 & 59 & 58 & - & 70 & 64 & 68 & 62 & 90 & & 82 & 62 & 62 \\
\hline \multirow[t]{2}{*}{13} & Pseudanabaena & 64 & 65 & 63 & 53 & 53 & - & 65 & 61 & 62 & 60 & 79 & 84 & & 62 & 47 \\
\hline & PCC7409 & 61 & 61 & 57 & 58 & 57 & - & 71 & 61 & 68 & 60 & 91 & 94 & & 59 & 58 \\
\hline \multirow[t]{2}{*}{14} & Porphyra & 60 & 60 & 60 & 58 & 52 & - & 61 & 54 & 61 & 56 & 65 & 66 & 63 & & 78 \\
\hline & purpurea & 56 & 56 & 53 & 56 & 56 & - & 72 & 55 & 72 & 54 & 69 & 67 & 66 & & 83 \\
\hline \multirow[t]{2}{*}{15} & Aglaothamnion & 65 & 64 & 32 & 60 & 60 & - & 61 & 54 & 65 & 55 & 64 & 65 & 64 & 78 & \\
\hline & neglectum & 57 & 57 & 53 & 53 & 53 & - & 71 & 53 & 71 & 53 & 64 & 64 & 63 & 82 & \\
\hline
\end{tabular}

Synechococcus cpeB genes (Table 1). Most notably, of the 14 base-pair changes between MIT9303 and MIT9313, all but one of these changes occurred in the third codon position. Comparable patterns were observed for the MIT9303 and MIT9313 cpeA genes (Table 1). Pairwise cpeB sequence comparisons among the low-light-adapted isolates (MIT9303, MIT9313, SS120, PAC1; Table 1) indicated that sequence identities ranged from $65 \%$ (MIT9303/MIT9313, PAC1) to $97 \%$ (MIT9303, MIT9313). However, cpeB of MED4 (highlight-adapted clade) exhibited $42 \%$ or less nucleotide identity with cpeB of other Prochlorococcus isolates (Table 1).

The number of synonymous $\left(K_{\mathrm{s}}\right)$ and nonsynonymous $\left(K_{\mathrm{a}}\right)$ differences for $c p e B$ and cpeA of Prochlorococcus as well as other cyanobacteria, and the $K_{\mathrm{a}} / K_{\mathrm{s}}$ ratios, are reported in Tables 2 and 3, respectively. For both $c p e B$ and cpeA, $K_{\mathrm{a}} / K_{\mathrm{s}}$ values were not elevated within the Prochlorococcus genus relative to marine Synechococcus (Table 3). Comparisons between the different genera, as well as between specific strains, indicated that the range of $K_{\mathrm{a}} / K_{\mathrm{s}}$ ratios was comparable (Table 3 ). Interestingly, while $K_{\mathrm{a}} / K_{\mathrm{s}}$ was consistently greater than one for cpeB, $K_{\mathrm{a}} / K_{\mathrm{s}}$ was less than one for $c p e A$, suggesting that the functional constraints on the evolution of these genes are different.

\section{$\mathbf{G}+\mathbf{C}$ base ratios}

Both the total and third codon $\mathrm{G}+\mathrm{C}$ base content of cpeB and cpeA were higher in MIT9303 and MIT9313 than in other Prochlorococcus isolates (Table 4). Among the six isolates that have been characterized thus far, the total G $+\mathrm{C}$ contents of $c p e B$ and $c p e A$ in MIT9303 and MIT9313 were closest to those of marine Synechococcus sp. For these two genes, the codon usage of MIT9303 and MIT9313 was shifted towards T at the third base position ( $\mathrm{T}>\mathrm{C}>\mathrm{G}$ or $\mathrm{A}$ ). This nucleotide frequency bias was similar to that of other Prochlorococcus isolates, whose $c p e B$ and $c p e A$ genes have third base positions favouring $T$ or $A(T>A>G \geqslant C)$. Similar nucleotide frequency biases towards $\mathrm{T}(\mathrm{T}>\mathrm{A}>\mathrm{C}$ or $\mathrm{G})$ have been reported previously for $p s b B$ and pet $B / D$ 
Table 2. Matrix of the number of synonymous $\left(K_{\mathrm{s}}\right)$ and nonsynonymous $\left(K_{\mathrm{a}}\right)$ differences for the cpe $B$ (above the diagonal) and cpeA (below the diagonal) genes of different cyanobacterial genera

Synonymous differences reported here have been normalized using the number of potential synonymous sites. Nonsynonymous differences have been normalized using the number of potential nonsynonymous sites. All values have been multiplied by 100 . The upper numbers (bold) indicate the number of synonymous substitutions; the lower numbers indicate the number of nonsynonymous substitutions.

\begin{tabular}{|c|c|c|c|c|c|c|c|c|c|c|}
\hline & & 1 & 2 & 3 & 4 & 5 & 6 & 7 & 8 & 9 \\
\hline \multirow[t]{2}{*}{1} & Pro & & $20 \cdot 4 \pm 4 \cdot 1$ & $18 \cdot 5 \pm 4 \cdot 1$ & $38 \cdot 3 \pm 5 \cdot 1$ & $24 \cdot 2 \pm 4 \cdot 3$ & $34 \cdot 9 \pm 4 \cdot 8$ & $26 \cdot 0 \pm 4 \cdot 4$ & $30 \cdot 8 \pm 4 \cdot 7$ & $24 \cdot 4 \pm 4 \cdot 4$ \\
\hline & MIT9303 & & $35 \cdot 5 \pm 3 \cdot 1$ & $35 \cdot 2 \pm 3 \cdot 1$ & $54 \cdot 0 \pm 3 \cdot 2$ & $33 \cdot 4 \pm 3 \cdot 1$ & $36 \cdot 1 \pm 3 \cdot 1$ & $36 \cdot 4 \pm 3 \cdot 1$ & $40 \cdot 0 \pm 3 \cdot 2$ & $41 \cdot 9 \pm 3 \cdot 2$ \\
\hline \multirow[t]{2}{*}{2} & Pro & $40 \cdot 2 \pm 4 \cdot 7$ & & $11 \cdot 4 \pm 3 \cdot 4$ & $29 \cdot 8 \pm 4 \cdot 9$ & $32 \cdot 0 \pm 4 \cdot 8$ & $34 \cdot 7 \pm 4 \cdot 9$ & $29 \cdot 1 \pm 4 \cdot 7$ & $29 \cdot 2 \pm 4 \cdot 7$ & $30 \cdot 9 \pm 4 \cdot 8$ \\
\hline & SS120 & $31 \cdot 4 \pm 2 \cdot 8$ & & $26 \cdot 6 \pm 2 \cdot 8$ & $45 \cdot 8 \pm 3 \cdot 2$ & $46 \cdot 0 \pm 3 \cdot 2$ & $47 \cdot 3 \pm 3 \cdot 2$ & $43 \cdot 6 \pm 3 \cdot 2$ & $47 \cdot 7 \pm 3 \cdot 2$ & $39 \cdot 9 \pm 3 \cdot 1$ \\
\hline \multirow[t]{2}{*}{3} & Pro PAC1 & $50 \cdot 6 \pm 4 \cdot 8$ & $43 \cdot 1 \pm 4 \cdot 9$ & & $35 \cdot 5 \pm 5 \cdot 2$ & $26 \cdot 0 \pm 4 \cdot 5$ & $29 \cdot 7 \pm 4 \cdot 8$ & $25 \cdot 0 \pm 4 \cdot 5$ & $22 \cdot 5 \pm 4 \cdot 4$ & $30 \cdot 0 \pm 4 \cdot 9$ \\
\hline & & $32 \cdot 4 \pm 2 \cdot 8$ & $24 \cdot 9 \pm 2 \cdot 6$ & & $46 \cdot 5 \pm 3 \cdot 1$ & $43 \cdot 2 \pm 3 \cdot 2$ & $47 \cdot 8 \pm 3 \cdot 2$ & $43 \cdot 8 \pm 3 \cdot 2$ & $45 \cdot 2 \pm 3 \cdot 2$ & $42 \cdot 2 \pm 3 \cdot 1$ \\
\hline \multirow[t]{2}{*}{4} & Pro MED4 & - & - & - & & $36 \cdot 4 \pm 5 \cdot 0$ & $42 \cdot 7 \pm 5 \cdot 2$ & $40 \cdot 1 \pm 5 \cdot 2$ & $45 \cdot 5 \pm 5 \cdot 2$ & $37 \cdot 2 \pm 5 \cdot 2$ \\
\hline & & - & - & - & & $58 \cdot 1 \pm 3 \cdot 2$ & $59 \cdot 7 \pm 3 \cdot 1$ & $56 \cdot 0 \pm 3 \cdot 2$ & $58 \cdot 6 \pm 3 \cdot 1$ & $50 \cdot 9 \pm 3 \cdot 2$ \\
\hline \multirow[t]{2}{*}{5} & Syn & $47 \cdot 0 \pm 4 \cdot 7$ & $56 \cdot 3 \pm 4 \cdot 7$ & $55 \cdot 1 \pm 4 \cdot 7$ & - & & $17 \cdot 4 \pm 3 \cdot 8$ & $12 \cdot 4 \pm 3 \cdot 3$ & $19 \cdot 7 \pm 4 \cdot 0$ & $17 \cdot 6 \pm 3 \cdot 9$ \\
\hline & $\begin{array}{l}\text { WH7803 } \\
\text { (PEI) }\end{array}$ & $33 \cdot 5 \pm 2 \cdot 8$ & $40 \cdot 0 \pm 2 \cdot 9$ & $38 \cdot 3 \pm 2 \cdot 9$ & - & & $33 \cdot 4 \pm 3 \cdot 1$ & $21 \cdot 0 \pm 2 \cdot 6$ & $36 \cdot 6 \pm 3 \cdot 1$ & $36 \cdot 0 \pm 3 \cdot 1$ \\
\hline \multirow[t]{2}{*}{6} & Syn & $52 \cdot 5 \pm 4 \cdot 7$ & $62 \cdot 7 \pm 4 \cdot 6$ & $64 \cdot 1 \pm 4 \cdot 6$ & - & $38 \cdot 6 \pm 4 \cdot 5$ & & $25 \cdot 0 \pm 4 \cdot 4$ & $13 \cdot 4 \pm 3 \cdot 4$ & $26.5 \pm 4.5$ \\
\hline & $\begin{array}{l}\text { WH8103 } \\
\text { (PEII) }\end{array}$ & $37 \cdot 8 \pm 2 \cdot 9$ & $44 \cdot 3 \pm 3 \cdot 0$ & $45 \cdot 2 \pm 3 \cdot 0$ & - & $31 \cdot 8 \pm 2 \cdot 8$ & & $34 \cdot 2 \pm 3 \cdot 1$ & $23 \cdot 1 \pm 2 \cdot 7$ & $44 \cdot 0 \pm 3 \cdot 2$ \\
\hline \multirow[t]{2}{*}{7} & Syn & $37 \cdot 3 \pm 4 \cdot 6$ & $50 \cdot 2 \pm 4 \cdot 8$ & $53 \cdot 9 \pm 4 \cdot 8$ & - & $34 \cdot 9 \pm 4 \cdot 4$ & $46.9 \pm 4 \cdot 6$ & & $20 \cdot 2 \pm 4 \cdot 1$ & $20 \cdot 7 \pm 4 \cdot 2$ \\
\hline & $\begin{array}{l}\text { WH8020 } \\
\text { (PEI) }\end{array}$ & $31 \cdot 3 \pm 2 \cdot 8$ & $38 \cdot 1 \pm 2 \cdot 9$ & $36 \cdot 3 \pm 2 \cdot 9$ & - & $14 \cdot 1 \pm 2 \cdot 1$ & $34 \cdot 2 \pm 2 \cdot 9$ & & $34 \cdot 1 \pm 3 \cdot 1$ & $33 \cdot 7 \pm 3 \cdot 0$ \\
\hline \multirow[t]{2}{*}{8} & Syn & $49 \cdot 9 \pm 4 \cdot 7$ & $55 \cdot 4 \pm 4 \cdot 8$ & $59 \cdot 6 \pm 4 \cdot 7$ & - & $47 \cdot 7 \pm 4 \cdot 6$ & $30 \cdot 1 \pm 4 \cdot 3$ & $46 \cdot 5 \pm 4 \cdot 7$ & & $22 \cdot 3 \pm 4 \cdot 3$ \\
\hline & $\begin{array}{l}\text { WH8020 } \\
\text { (PEII) }\end{array}$ & $42 \cdot 1 \pm 3 \cdot 0$ & $42 \cdot 6 \pm 2 \cdot 9$ & $43 \cdot 4 \pm 3 \cdot 0$ & - & $31 \cdot 7 \pm 2 \cdot 8$ & $12 \cdot 2 \pm 2 \cdot 0$ & $36 \cdot 2 \pm 2 \cdot 9$ & & $41 \cdot 0 \pm 3 \cdot 2$ \\
\hline \multirow[t]{2}{*}{9} & Syn & $50 \cdot 1 \pm 4 \cdot 7$ & $51 \cdot 4 \pm 4 \cdot 8$ & $52 \cdot 2 \pm 4 \cdot 8$ & - & $46 \cdot 6 \pm 4 \cdot 6$ & $54 \cdot 8 \pm 4 \cdot 6$ & $44 \cdot 4 \pm 4 \cdot 6$ & $44 \cdot 4 \pm 4 \cdot 7$ & \\
\hline & PCC6701 & $34 \cdot 1 \pm 2 \cdot 8$ & $39 \cdot 9 \pm 2 \cdot 9$ & $37 \cdot 0 \pm 2 \cdot 9$ & - & $27 \cdot 4 \pm 2 \cdot 7$ & $32 \cdot 3 \pm 2 \cdot 8$ & $30 \cdot 5 \pm 2 \cdot 8$ & $31 \cdot 7 \pm 2 \cdot 8$ & \\
\hline
\end{tabular}

Table 3. Ratios of the number of nonsynonymous to synonymous differences $\left(K_{\mathrm{a}} / K_{\mathrm{s}}\right)$ in the $с р е B$ (above the diagonal) and $c p e A$ (below the diagonal) sequences of different cyanobacteria

\begin{tabular}{|c|c|c|c|c|c|c|c|c|c|c|}
\hline & & 1 & 2 & 3 & 4 & 5 & 6 & 7 & 8 & 9 \\
\hline 1 & $\begin{array}{l}\text { Prochlorococcus } \\
\text { MIT9303 }\end{array}$ & & 1.74 & 1.90 & 1.41 & 1.38 & $1 \cdot 03$ & $1 \cdot 4$ & $1 \cdot 3$ & $1 \cdot 72$ \\
\hline 2 & $\begin{array}{l}\text { Prochlorococcus } \\
\text { SS120 }\end{array}$ & $0 \cdot 78$ & & $2 \cdot 33$ & 1.54 & 1.44 & $1 \cdot 36$ & $1 \cdot 50$ & $1 \cdot 63$ & 1.29 \\
\hline 3 & $\begin{array}{l}\text { Prochlorococcus } \\
\text { PAC1 }\end{array}$ & 0.64 & 0.58 & & $1 \cdot 31$ & 1.66 & $1 \cdot 61$ & $1 \cdot 75$ & $2 \cdot 01$ & $1 \cdot 41$ \\
\hline 4 & $\begin{array}{l}\text { Prochlorococcus } \\
\text { MED4 }\end{array}$ & - & - & - & & $1 \cdot 60$ & $1 \cdot 40$ & $1 \cdot 40$ & $1 \cdot 29$ & 1.37 \\
\hline 5 & $\begin{array}{l}\text { Synechococcus } \\
\text { WH7803 (PEI) }\end{array}$ & $0 \cdot 71$ & $0 \cdot 71$ & $0 \cdot 70$ & - & & 1.92 & $1 \cdot 69$ & $1 \cdot 86$ & $2 \cdot 05$ \\
\hline 6 & $\begin{array}{l}\text { Synechococcus } \\
\text { WH8103 (PEII) }\end{array}$ & $0 \cdot 72$ & $0 \cdot 71$ & $0 \cdot 71$ & - & 0.82 & & $1 \cdot 37$ & $1 \cdot 72$ & $1 \cdot 66$ \\
\hline 7 & $\begin{array}{l}\text { Synechococcus } \\
\text { WH8020 (PEI) }\end{array}$ & $0 \cdot 84$ & $0 \cdot 76$ & 0.67 & - & 0.40 & 0.73 & & 1.69 & $1 \cdot 63$ \\
\hline 8 & $\begin{array}{l}\text { Synechococcus } \\
\text { WH8020 (PEII) }\end{array}$ & $0 \cdot 84$ & $0 \cdot 77$ & 0.73 & - & 0.66 & $0 \cdot 41$ & $0 \cdot 78$ & & $1 \cdot 84$ \\
\hline 9 & $\begin{array}{l}\text { Synechocystis } \\
\text { PCC6701 }\end{array}$ & 0.68 & $0 \cdot 78$ & $0 \cdot 71$ & - & 0.59 & 0.59 & 0.69 & $0 \cdot 71$ & \\
\hline
\end{tabular}


Table 4. $\mathrm{G}+\mathrm{C}$ base ratios of $c p e B$ and $c p e A$ of Prochlorococcus (Pro) and marine Synechococcus (Syn)

\begin{tabular}{|c|c|c|c|c|}
\hline \multirow[t]{2}{*}{ Strain } & \multicolumn{2}{|c|}{$\mathrm{Mol} \% \mathrm{G}+\mathrm{C}$ сpeB } & \multicolumn{2}{|c|}{$\mathrm{Mol} \% \mathrm{G}+\mathrm{C}$ cpeA } \\
\hline & Total & 3rd codon & Total & 3rd codon \\
\hline Pro MIT9303 & 53 & 49 & 49 & 43 \\
\hline Pro MIT9313 & 53 & 49 & 49 & 42 \\
\hline Pro SS120 & 37 & 17 & 40 & 30 \\
\hline Pro PAC1 & 43 & 28 & 38 & 24 \\
\hline Pro PAC2 & 41 & 20 & 37 & 23 \\
\hline Pro MED4 & 28 & 17 & - & - \\
\hline Syn WH7803 (PEI) & 61 & 72 & 60 & 66 \\
\hline Syn WH8103 (PEII) & 60 & 69 & 60 & 70 \\
\hline Syn WH8020 (PEI) & 54 & 50 & 54 & 49 \\
\hline Syn WH8020 (PEII) & 57 & 57 & 54 & 52 \\
\hline
\end{tabular}

genes of several Prochlorococcus isolates, including SS120 and NATL2A (Urbach et al., 1998; Partensky et al., 1999), and mutational biases may be a cause of these observed patterns.

\section{Derived $\beta$-PE and $\alpha$-PE amino acid sequences}

As indicated in Table 1, Prochlorococcus $\beta$-PE sequences (excluding MED4) were consistently more similar to each other (77-99\% identity) than they were to the $\beta$ PEs of marine Synechococcus strains (60-67\% identity), or other cyanophytes and rhodophytes. Pairwise comparisons between MIT9303/MIT9313 and SS120 revealed that this was most likely because the majority of differences were in the third codon position and many were synonymous. The derived $\beta$-PE sequence of MED4 exhibited the lowest amount $(\leqslant 41 \%)$ of sequence identity with the $\beta$-PE sequences of the other isolates (Table 1). Similar patterns were observed for the $\alpha$-PE sequences of Prochlorococcus (Table 1).

Both the derived $\beta$-PE sequences and $\alpha$-PE sequences exhibited $99 \%$ amino acid identity between MIT9303 and MIT9313 (Table 1). While the single amino acid residue difference between $\beta$-PE of MIT9303 and MIT9313 involved the conserved substitution of Asp144 (MIT9303) and Asn-144 (MIT9313), the single residue difference between $\alpha$-PE involved the semiconserved substitution of Ala-29 (MIT9303) and Val-29 (MIT9313).

Table 5. Nonparametric relative rate test scores for the $\beta$-PE and $\alpha$-PE sequences of Prochlorococcus and other cyanobacteria

\begin{tabular}{|c|c|c|c|c|}
\hline A & B & C (outgroup) & $x^{2} \dagger$ & $\rho \dagger$ \\
\hline Prochlorococcus MIT9303 & Prochlorococcus SS120 & Synechococcus WH7803(PEI) & $\begin{array}{l}1.38 \\
1.96\end{array}$ & $\begin{array}{l}\mathbf{0} \cdot 239 \\
0 \cdot 162\end{array}$ \\
\hline Prochlorococcus MIT9303 & Prochlorococcus SS120 & Synechocystis PCC6701 & $\begin{array}{l}\mathbf{1} \cdot 64 \\
0.62\end{array}$ & $\begin{array}{l}0 \cdot 201 \\
0 \cdot 433\end{array}$ \\
\hline Prochlorococcus MED4 & Prochlorococcus MIT9303 & Synechococcus WH7803(PEI) & $\begin{array}{c}40 \cdot 50 \\
-\end{array}$ & $\begin{array}{c}0.00 * \\
-\end{array}$ \\
\hline Prochlorococcus MED4 & Prochlorococcus MIT9303 & Synechocystis PCC6701 & $\begin{array}{c}37 \cdot 70 \\
-\end{array}$ & $\begin{array}{c}0 \cdot 00^{*} \\
-\end{array}$ \\
\hline Prochlorococcus MED4 & Prochlorococcus SS120 & Synechococcus WH7803(PEI) & $\begin{array}{c}35 \cdot 06 \\
-\end{array}$ & $\begin{array}{c}0.00^{*} \\
-\end{array}$ \\
\hline Prochlorococcus MED4 & Prochlorococcus SS120 & Synechocystis PCC6701 & $\begin{array}{c}33 \cdot 38 \\
-\end{array}$ & $\begin{array}{l}0.00^{*} \\
-\end{array}$ \\
\hline Prochlorococcus MIT9303 & Synechococcus WH8020 (PEI) & Calothrix PCC7601 & $\begin{array}{l}\mathbf{9} \cdot 26 \\
5 \cdot 76\end{array}$ & $\begin{array}{l}\mathbf{0 . 0 0 2 *} \\
0 \cdot 016^{*}\end{array}$ \\
\hline Prochlorococcus MIT9303 & Synechococcus WH8020 (PEII) & Calothrix PCC7601 & $\begin{array}{l}\mathbf{0} \cdot 00 \\
0 \cdot 69\end{array}$ & $\begin{array}{l}\mathbf{1} \cdot 00 \\
0 \cdot 405\end{array}$ \\
\hline Prochlorococcus SS120 & Synechococcus WH8020 (PEI) & Calothrix PCC7601 & $\begin{array}{r}20 \cdot 45 \\
8 \cdot 80\end{array}$ & $\begin{array}{l}\mathbf{0} \cdot 00^{*} \\
0 \cdot 003^{*}\end{array}$ \\
\hline Prochlorococcus SS120 & Synechococcus WH8020 (PEII) & Calothrix PCC7601 & $\begin{array}{l}\mathbf{3} \cdot \mathbf{6 0} \\
2 \cdot 37\end{array}$ & $\begin{array}{l}\mathbf{0} \cdot 058 \\
0 \cdot 123\end{array}$ \\
\hline Prochlorococcus MED4 & Synechococcus WH8020 (PEI) & Calothrix PCC7601 & $\begin{array}{c}56 \cdot 82 \\
-\end{array}$ & $\begin{array}{c}0.00^{*} \\
-\end{array}$ \\
\hline Prochlorococcus MED4 & Synechococcus WH8020 (PEII) & Calothrix PCC7601 & $\begin{array}{c}38.63 \\
-\end{array}$ & $\begin{array}{c}0 \cdot 00^{*} \\
-\end{array}$ \\
\hline $\begin{array}{l}\text { Synechococcus WH8020 } \\
\text { (PEI) }\end{array}$ & Synechococcus WH8020 (PEII) & Calothrix PCC7601 & $\begin{array}{l}8 \cdot 10 \\
2 \cdot 47\end{array}$ & $\begin{array}{l}\mathbf{0 . 0 0 4 *} \\
0 \cdot 116\end{array}$ \\
\hline
\end{tabular}

†The upper numbers (bold) represent $\chi^{2}$ or $\rho$ for $\beta$-PE analyses; the lower numbers represent $\chi^{2}$ or $\rho$ for $\alpha$-PE analyses. Low $p$ values $(<0.05)$ are marked with an asterisk. 
(a) MIT9303

MIT9313

SS120

MED 4

WH7803C-PEI

WH8103C-PEII

WH8020-PEI

WH8020-PEII

Calothrix

MIT9303

MIT9313

SS120

MED4

WH7803C-PEI

WH8103C-PEII

WH8020-PEI

WH8020-PEII

Calothrix

MIT9303

MIT9313

SS120

MED 4

WH7803C-PEI

WH8103C-PEII

WH8020-PEI

WH8020-PEII

Calothrix

(b) MIT9303

MIT 9313

SS120

WH7803C-PEI

WH8103C-PEII

WH8020-PEI

WH8020-PEII

Calothrix

MIT 9303

MIT9313

SS120

WH7803C-PEI

WH8103C-PEII

WH8020-PEI

WH8020-PEII

Calothrix

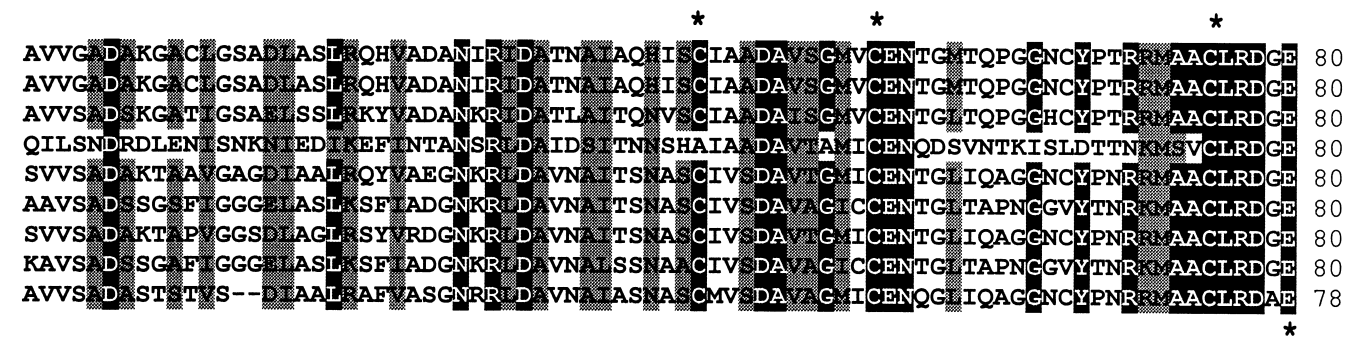

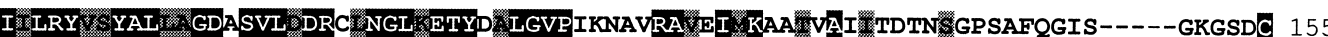

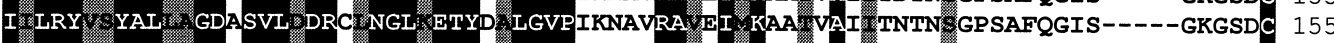

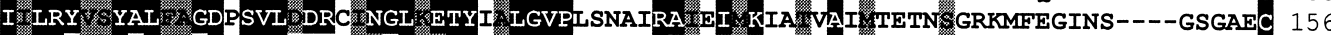

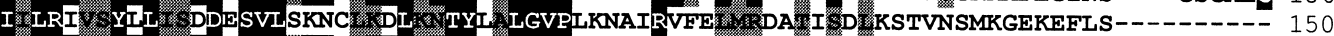

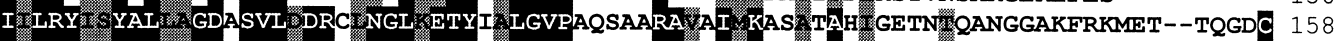
IVLRY SYAL IA GDA SVLODRCENGL ETYA LGVPTGSASRA VAI KAA GALITNTNSQPKKMPVT-------TGDC 152

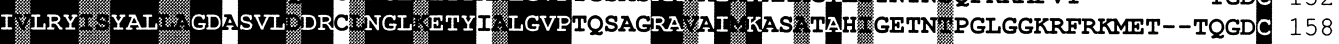

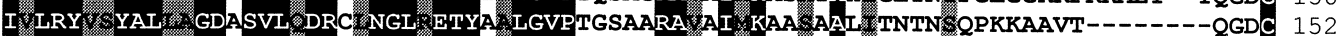

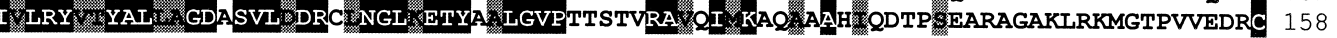
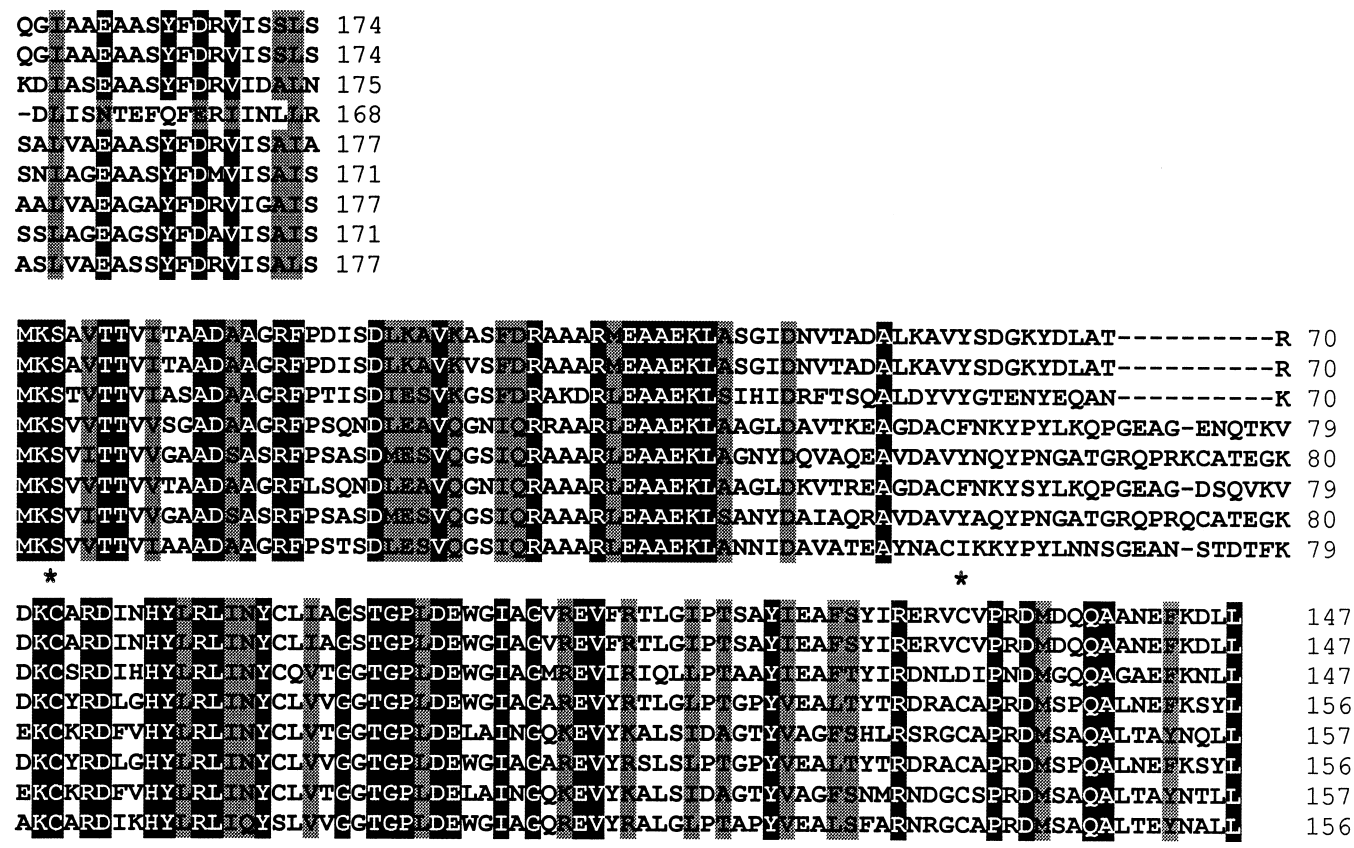

Fig. 2. Alignment of deduced amino acid sequences of $\beta$-PE (a) and $\alpha$-PE (b) of Prochlorococcus isolates MIT9303, MIT9313, SS120 and MED4 ( $\beta$-PE only), marine Synechococcus sp. strains WH7803, WH8103 and WH8020, and Calothrix sp. strain PCC7601 (formerly Fremyella diplosiphon). Identical residues are presented in white type on a black background. Black type on grey squares indicates highly conserved substitutions (within the groups STA NEQK NHQK NDEQ QHRK MILV MILF HY FYW). Putative chromophore-binding sites are indicated with an asterisk above the corresponding Cys residue.

Within the Prochlorococcus lineage, relative rate tests for $\beta$ - and $\alpha$-PE revealed that mutations in these sequences appear to be accumulating at approximately the same rate for low-light-adapted strains such as MIT9303 and SS120 ( $\chi^{2}$ was not significant at the $5 \%$ level) (Table 5). However, the rate at which the $\beta$-PE sequence is evolving in MED4 is not the same as that in MIT9303 or SS120. Instead, relative rate tests suggested an elevated rate of evolution for the high-light-adapted MED4 sequence (Table 5).

The results of the relative rate tests between Prochlorococcus and Synechococcus PE sequences depended on whether Synechococcus PE (I) or (II) was used in the actual test (Table 5). Differences in the rate at which mutations have accumulated in the PE sequences of Prochlorococcus strains MIT9303/SS120 and the PE (I) sequence of Synechococcus WH8020 were significant (Table 5). However, relative rate tests suggested that mutations have accumulated at approximately the same rate in the Prochlorococcus strain MIT9303/SS120 PE sequences as in the Synechococcus PE (II) sequences. In the case of MED4, all relative rate tests with Synechococcus suggested that the rate at which $\beta$-PE is evolving is not equivalent, and that mutations have accumulated faster in MED4 (Table 5). 

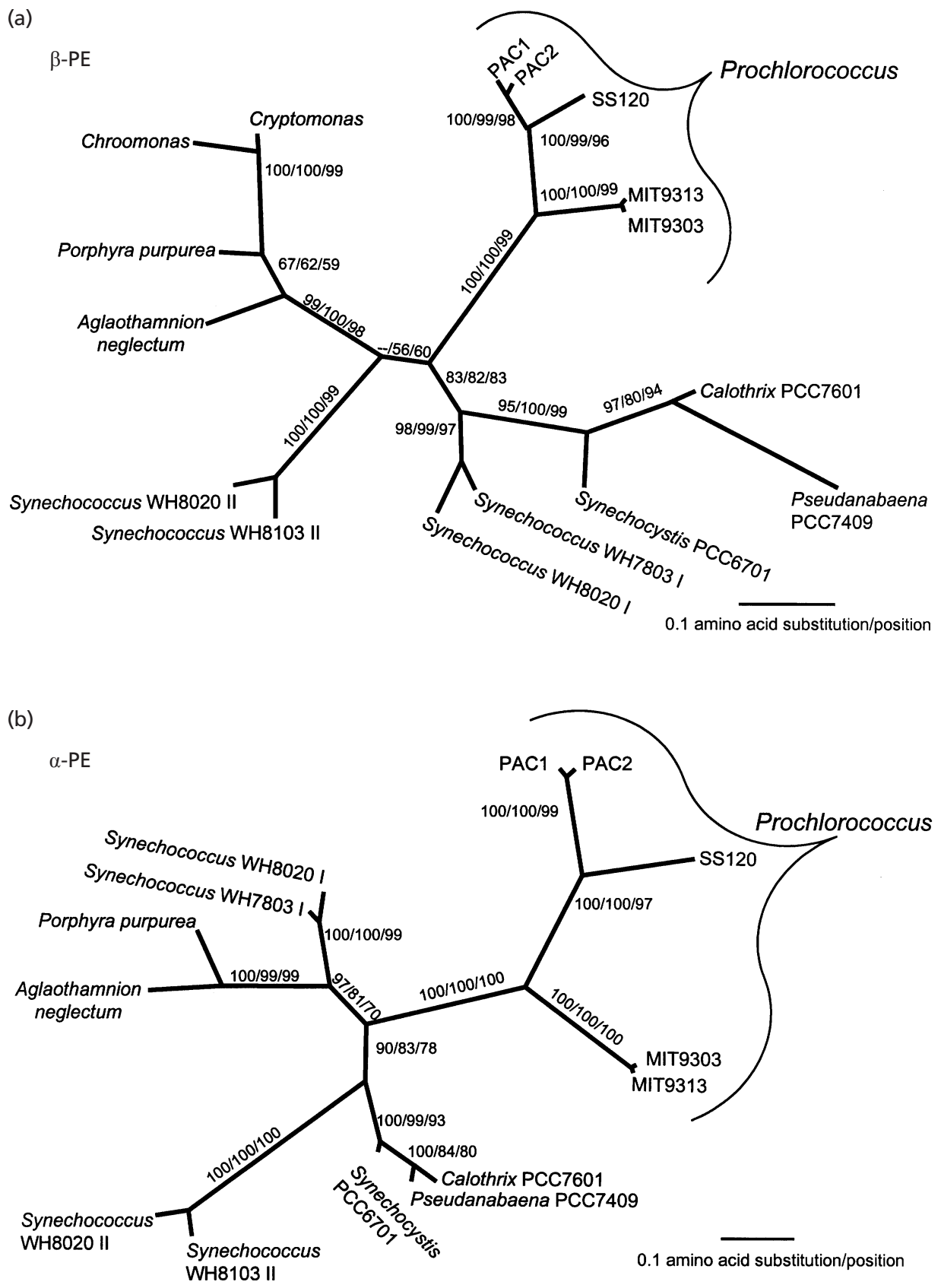

Fig. 3. Phylogenetic analysis of PE proteins from Prochlorococcus, other members of the cyanobacterial lineage, red algae and cryptomonads, using maximum-likelihood, distance and parsimony methods. Likelihood trees are shown for both $\beta$-PE (a) and $\alpha$-PE (b); identical tree topologies were obtained in distance and parsimony analyses. Support values for internal branches (percentage support from quartet puzzling for likelihood or percentage bootstrap values from 1000 resamplings for distance and parsimony analyses) are displayed at each node in the following order: likelihood/distance/parsimony.

\section{Identification of putative functional residues}

Alignments of the derived amino acid sequences of the $\beta$ PE subunit of MIT9303 and MIT9313 indicated that many residues known for their functional importance in the $\beta$-PE subunits of other organisms (Apt et al., 1995) were conserved in all of the Prochlorococcus sequences, excluding MED4 (Fig. 2a, b). These included residues that serve as putative chromophore attachment sites (Cys-50, Cys-61, Cys-82, Cys-162), function in chromophore interaction and stabilization (Arg-77, Arg-78, Ala-80/81, Arg-84, Asp-85), and have a role in subunit interactions (Asp-13, Arg-91, Tyr-92, Tyr-95, Arg-108) (Apt et al., 1995). In contrast, the MED4 $\beta$-PE sequence 


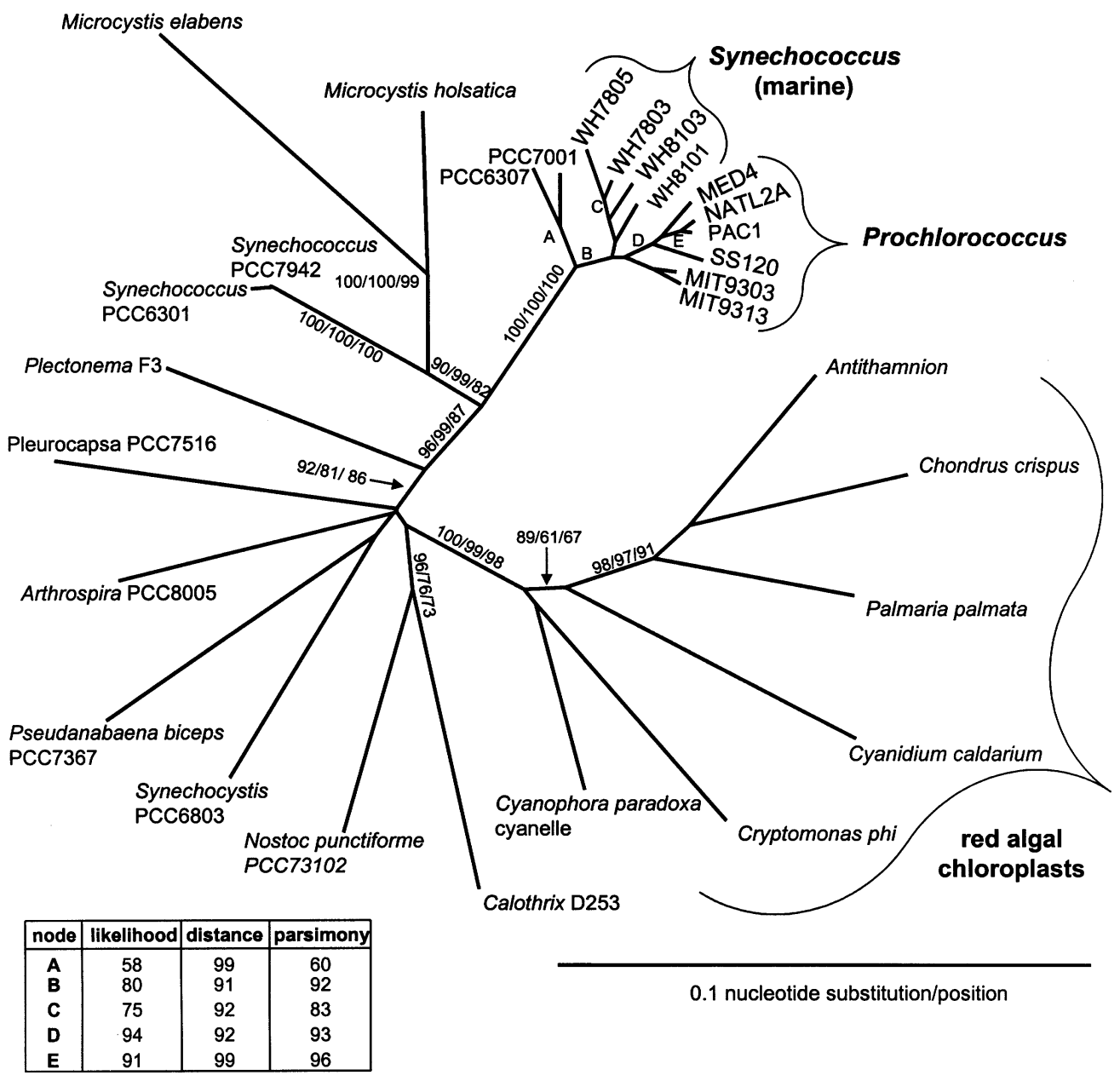

Fig. 4. Phylogenetic relationships among Prochlorococcus and other organisms based on 165 rDNA sequences. The distance tree is shown with bootstrap values for all three methods displayed in the following order: likelihood/minimum evolution-distance/parsimony.

lacked several of these highly conserved residues, including Cys-50(43), Cys-162(155), Ala-80(73)/81(74) and Tyr-92(85) (Fig. 2a).

Alignment of the derived $\alpha$-PE sequences indicated that several residues important in chromophore attachment or protein interactions were also conserved in this subunit (Fig. 2b). Most notably, both MIT9303 and MIT9313 possessed a cysteine residue at position 130 that is highly conserved among other cyanobacteria and red algae and functions in chromophore binding (Apt et al., 1995). However, this Cys-130 residue is not conserved in the $\alpha$-PE sequences of any other Prochlorococcus isolate (Fig. 2b). In SS120, this position contains an aspartic acid residue (Hess et al., 1996), which does not represent a conserved substitution.

\section{Phylogenetic analyses}

Phylogenetic trees constructed using protein sequences deduced from $c p e B$ and $c p e A$ genes (Fig. 3a, b) revealed that the position of the Prochlorococcus cluster relative to marine Synechococcus was not congruent with $16 \mathrm{~S}$ rDNA based phylogenetic groupings (Fig. 4). Prochlorococcus $\beta$-PE and $\alpha$-PE sequences did not group with either type I or type II $\beta$-PE and $\alpha$-PE sequences from marine Synechococcus (Fig. 3a, b). However, within the Prochlorococcus limb, the clustering of isolates based on both $\beta$ - and $\alpha$-PE sequences was consistent with their positions in the $16 \mathrm{~S} \mathrm{rDNA}$-based tree. As in ribosomal trees, MIT9303 and MIT9313 are very closely related (Table 6, Fig. 4) and form the basal branch of the Prochlorococcus clade. Similar tree topologies and levels of support were obtained in trees rooted using phycocyanin sequences and in trees constructed using $c p e B$ and cpeA nucleotide sequences (both all positions and only the first two codon positions) (data not shown). The MED4 $\beta$-PE sequence was not included in the tree (Fig. 3a) because it is highly degenerate and shared low sequence identity $(\leqslant 36 \%)$ with other cyanobacterial and red algal $\beta$-PEs (Table 1). This resulted in its branch length being five times longer than any of the others in the tree. In phylogenetic trees, the placement of such 
Table 6. 16S rDNA sequence identity matrix for Prochlorococcus (Pro) and Synechococcus (Syn)

\begin{tabular}{|c|c|c|c|c|c|c|c|c|}
\hline & 2 & 3 & 4 & 5 & 6 & 7 & 8 & 9 \\
\hline Pro MIT9303 & $98 \cdot 97$ & $97 \cdot 47$ & $97 \cdot 28$ & $97 \cdot 19$ & $97 \cdot 00$ & $98 \cdot 41$ & $98 \cdot 23$ & $97 \cdot 75$ \\
\hline 2 Pro MIT9313 & & $97 \cdot 94$ & $98 \cdot 13$ & $98 \cdot 03$ & $97 \cdot 66$ & $97 \cdot 75$ & $97 \cdot 19$ & $97 \cdot 28$ \\
\hline 3 Pro SS120 & & & $98 \cdot 32$ & $98 \cdot 03$ & $98 \cdot 32$ & $97 \cdot 57$ & $97 \cdot 00$ & $97 \cdot 29$ \\
\hline 4 Pro PAC1 & & & & $99 \cdot 53$ & $98 \cdot 78$ & $97 \cdot 19$ & $96 \cdot 72$ & $96 \cdot 91$ \\
\hline 5 Pro NATL2A & & & & & $98 \cdot 50$ & $96 \cdot 91$ & $96 \cdot 44$ & $96 \cdot 82$ \\
\hline 6 Pro MED4 & & & & & & $97 \cdot 00$ & $96 \cdot 72$ & $96 \cdot 91$ \\
\hline 7 Syn WH7803 & & & & & & & $98 \cdot 60$ & $98 \cdot 13$ \\
\hline 8 Syn WH8103 & & & & & & & & $98 \cdot 41$ \\
\hline 9 Syn WH8101 & & & & & & & & \\
\hline
\end{tabular}

long branches is often at odds with true evolutionary position (Swofford et al., 1996).

\section{DISCUSSION}

$16 \mathrm{~S}$ rDNA-based phylogenetic analyses suggest that Prochlorococcus and marine Synechococcus share a common, phycobiliprotein-containing ancestor, and may have both diversified at a similar point in time (Urbach et al., 1998). Although Prochlorococcus has evolved to use a chlorophyll $a_{2} / b_{(2)}$ light-harvesting complex as its major antenna, it still retains genes encoding the phycobiliprotein PE (Hess et al., 1996; LaRoche et al., 1996; Ting et al., 1999). The branching order we observed among Prochlorococcus PE genes (not including the degenerate MED4 cpeB) was congruent with previous $16 \mathrm{~S}$ rDNA sequence analyses for this genus. Furthermore, both complete sequence and third codon $\mathrm{G}+\mathrm{C}$ base frequencies of $c p e B$ and $c p e A$ were correlated with $16 \mathrm{~S}$ rDNA phylogenetic tree branching patterns, with isolates such as MIT9303 and MIT9313 exhibiting the highest $(0.48-0.53) \mathrm{G}+\mathrm{C}$ base content ratios for these particular genes. A similar correlation between third codon position $\mathrm{G}+\mathrm{C}$ base frequencies and the branching patterns of $16 \mathrm{~S}$ rRNA gene phylogenetic trees was demonstrated previously for several Prochlorococcus and Synechococcus genes (Urbach et al., 1998; Rocap, 2000). In particular, other G + C-rich genes identified in MIT9303 include $p s b B(0.52)$, pet $B$ (0.58) and petD (0.58) (Urbach et al., 1998). Although these data support the idea that the PE genes of Prochlorococcus were unlikely to have been acquired by lateral gene transfer (Hess et al., 1999), they do not completely rule out the possibility of recent horizontal transfer from a close relative, particularly in the case of MED4. Nevertheless, no MED4-like cpeB sequence has been previously described in any other photosynthetic organism. Thus it is most parsimonious to presume that the PE genes were retained from the phycobilisomecontaining ancestor of Prochlorococcus and Synechococcus.

Our sequence analyses suggest that within the Prochlorococcus lineage, the selective forces shaping the evolution of the PE gene set have not been uniform. Notably, this is apparent in the unique loss of the gene encoding the $\alpha$ PE subunit in the MED4 strain compared to more deeply branching isolates such as MIT9303 and MIT9313. Loss of PE genes has been suggested to have occurred in other cyanobacterial lines (Apt et al., 1995). MIT9303 and MIT9313 have the highest degree of 16S rDNA sequence similarity to marine Synechococcus relative to other cultured Prochlorococcus isolates (Table 6), and only they have retained a highly conserved putative chromophore binding site (Cys-130) in their $\alpha$-PE subunit. Furthermore, relative rate tests suggest that mutations have not accumulated at an equivalent rate in the $\beta$-PE sequences of Prochlorococcus. In MED4, the presence of a degenerate form of $\beta$-PE, characterized by several point mutations and deletions at highly conserved sites, is consistent with an elevated rate of evolution. In addition, the ratios of nonsynonymous $\left(K_{\mathrm{a}}\right)$ to synonymous $\left(K_{\mathrm{s}}\right)$ substitutions were not elevated in MED4 relative to other Prochlorococcus or Synechococcus strains. Thus, even though MED4 lacks the gene encoding the $\alpha$-PE subunit, these results suggest that its $c p e B$ gene is still under selection, although likely a different type of selection than in other Prochlorococcus strains. As a high-light-adapted strain of Prochlorococcus, MED4 is thought to have arisen more recently than its low-light-adapted counterparts (e.g. MIT9303, MIT9313, SS120, PAC1) (Urbach et al., 1998; Rocap, 2000), and thus could have been subjected to different selective pressures (i.e. light and/or nutrient availability), leading to dissimilar genetic recombination and/or deletion events.

Although $c p e B$ and/or cpeA expression has been detected in SS120 (Hess et al., 1996, 1999), and in MIT9303 and MIT9313 (C. Ting, unpublished results), the exact role of PE in the Prochlorococcus genus remains to be established. PE is clearly not the major constituent of the light-harvesting apparatus in any extant Prochlorococcus strain (Chisholm et al., 1992; Goericke \& Repeta, 1992; LaRoche et al., 1996; Partensky et al., 1997), as it is in Synechococcus (Ong \& Glazer, 1991; Wilbanks et al., 1991). In SS120, the PE genes are part of a larger cluster, within which other phycobiliprotein-related genes (cpeZ, cpeY, mpeX, 
ppeC) can be found (Hess et al., 1999). But there is no evidence that PE forms part of a cyanobacterial-like phycobilisome structure in Prochlorococcus (Chisholm et al., 1988; Fields et al., 1997; C. S. Ting and others, unpublished). It remains to be explored whether the phycobiliproteins of Prochlorococcus are located within the intracytoplasmic lamellar space, as in cryptophytes (Gantt et al., 1971).

Our phylogenetic analyses based on PE sequences show that the position of the Prochlorococcus cluster relative to Synechococcus was not congruent with $16 \mathrm{~S}$ rDNAbased trees. Additional analyses indicated that the ratios of nonsynonymous $\left(K_{\mathrm{a}}\right)$ to synonymous $\left(K_{\mathrm{s}}\right)$ substitutions for the $c p e B$ and $c p e A$ genes were not elevated in Prochlorococcus relative to Synechococcus. Instead, $K_{\mathrm{a}} / K_{\mathrm{s}}$ ratios were similar between these genera. This suggests that the sequence differences we observed may be due to elevated mutation rates rather than relaxed selection. This is consistent with the results of relative rate tests between Prochlorococcus $\beta / \alpha-\mathrm{PE}$ and Synechococcus $\beta / \alpha$-PE (I) sequences. Thus although PE has a different function in Prochlorococcus, these results suggest that the $c p e B$ and $c p e A$ genes are still under selection, albeit a different type of selection than in Synechococcus.

Future work on the role of PE in Prochlorococcus may help to identify whether this protein confers a biological advantage under specific environmental conditions. However, with the change in selection on the PE genes we have observed within the Prochlorococcus lineage, it is unlikely that the function of PE will be conserved among all strains. Comparisons between Prochlorococcus and Synechococcus provide a striking example of how the evolution of a key protein complex, the lightharvesting antenna system, has proceeded along very different paths in two globally important marine prokaryotes.

\section{ACKNOWLEDGEMENTS}

We would like to thank John Waterbury (Dept of Biology, Woods Hole Oceanographic Institution) for kindly providing us with Synechococcus sp. strain WH8103, Mitchell Sogin for the use of the LICOR model 4000L Sequencer at the Marine Biological Laboratory (Woods Hole, MA) and the DOE Joint Genome Institute for use of preliminary sequence data (http://www.jgi.doe.gov/JGI_microbial/html/). Special thanks are also extended to Thomas Owens and Wolfgang Hess for their critical reading of the manuscript. This study was supported by a NSF Postdoctoral Research Fellowship in Biosciences Related to the Environment (C.S.T.), by a NSF Graduate Student Fellowship (G.R.), by NSF OCE-9820035 (S. W.C.), and by NASA (NAG5-3727) (G.R., S. W.C.).

\section{REFERENCES}

Apt, K. E., Collier, J. L. \& Grossman, A. R. (1995). Evolution of the phycobiliproteins. J Mol Biol 248, 79-96.

Ausubel, F., Brent, R., Kingston, R. E., Moore, D. D., Seidman, J. G., Smith, J. A. \& Struhl, K. (1995). Short Protocols in Molecular Biology, 3rd edn. New York: Wiley.
Borodovsky, M. \& McIninch, J. (1993). GeneMark: parallel gene recognition for both DNA strands. Comp Chem 17, 123-133.

Bryant, D. A. (1991). Cyanobacterial phycobilisomes: progress toward complete structural and functional analysis via molecular genetics. In The Photosynthetic Apparatus: Molecular Biology and Operation, pp. 257-300. Edited by L. Bogorad \& K. Vasil. Boston, MA: Academic Press.

Chisholm, S. W., Olson, R. J., Zettler, E. R., Waterbury, J., Goericke, R. \& Welschmeyer, N. (1988). A novel free-living prochlorophyte occurs at high cell concentrations in the oceanic euphotic zone. Nature 334, 340-343.

Chisholm, S. W., Frankel, S. L., Goericke, R., Olson, R. J., Palenik, B., Waterbury, J. B., West-Johnsrud, L. \& Zettler, E. (1992). Prochlorococcus marinus nov. gen. nov. sp.: an oxyphototrophic marine prokaryote containing divinyl chlorophyll $a$ and $b$. Arch Microbiol 157, 297-300.

Felsenstein, J. (1989). PHYLIP-phylogeny inference package (version 3.2). Cladistics 5, 164-166.

Fields, S. D., Strout, G. W. \& Russell, S. D. (1997). Spray-freezing and freeze substitution (SFFS) of cell suspensions for improved preservation of ultrastructure. Microsc Res Tech 38, 315-328.

Gantt, E., Edwards, M. R. \& Provasoli, L. (1971). Chloroplast structure of the Cryptophyceae. Evidence for phycobiliproteins within intrathylakoidal spaces. J Cell Biol 48, 280-290.

Goericke, R. \& Repeta, D. J. (1992). The pigments of Prochlorococcus marinus: the presence of divinyl chlorophyll $a$ and $b$ in a marine procaryote. Limnol Oceanogr 37, 425-433.

Hess, W. R., Weihe, A., Loiseaux-de Goer, S., Partensky, F. \& Vaulot, D. (1995). Characterization of the single $p s b A$ gene of Prochlorococcus marinus CCMP 1375 (Prochlorophyta). Plant Mol Biol 27, 1189-1196.

Hess, W. R., Partensky, F., van der Staay, G. W. M., GarciaFernandez, J. M., Borner, T. \& Vaulot, D. (1996). Coexistence of phycoerythrin and a chlorophyll $a / b$ antenna in a marine prokaryote. Proc Natl Acad Sci US A 93, 11126-11130.

Hess, W. R., Steglich, C., Lichtle, C. \& Partensky, F. (1999). The phycoerythrins of Prochlorococcus marinus are associated to the thylakoid membrane and are encoded by a single large gene cluster. Plant Mol Biol 40, 507-521.

Ina, Y. (1995). New methods for estimating the numbers of synonymous and nonsynonymous substitutions. J Mol Evol 40, 190-226.

Kumar, S., Tamura, K., Jakobsen, I. B. \& Nei, M. (2001). MEGA2: molecular evolutionary genetics analysis software. Bioinformatics (in press).

Lake, J. A. (1994). Reconstructing evolutionary trees from DNA and protein sequences: paralinear distances. Proc Natl Acad Sci US A 91, 1455-1459.

LaRoche, J., van der Staay, G. W. M., Partensky, F. \& 8 other authors (1996). Independent evolution of the prochlorophyte and green plant chlorophyll $a / b$ light-harvesting proteins. Proc Natl Acad Sci U S A 93, 15244-15248.

Maidak, B. L., Cole, J. R., Lilburn, T. G. \& 12 other authors (2000). The RDP (Ribosomal Database Project) continues. Nucleic Acids Res 28, 173-174.

Moore, L. R. \& Chisholm, S. W. (1999). Photophysiology of the marine cyanobacterium Prochlorococcus: ecotypic differences among cultured isolates. Limnol Oceanogr 44, 628-638.

Moore, L. R., Rocap, G. \& Chisholm, S. W. (1998). Physiology and molecular phylogeny of coexisting Prochlorococcus ecotypes. Nature 393, 464-467. 
Nei, M. \& Gojobori, T. (1986). Simple methods for estimating the numbers of synonymous and nonsynonymous nucleotide substitutions. Mol Biol Evol 3, 418-426.

Nei, M. \& Kumar, S. (2000). Molecular Evolution and Phylogenetics. New York: Oxford University Press.

Ong, L. J. \& Glazer, A. N. (1991). Phycoerythrins of marine unicellular cyanobacteria. I. Bilin types and locations and energy transfer pathways in Synechococcus spp. phycoerythrins. J Biol Chem 266, 9515-9527.

Page, R. D. M. (1996). TREEVIEW: an application to display phylogenetic trees on personal computers. Comput Appl Biosci 12, 357-358.

Palenik, B. \& Haselkorn, R. (1992). Multiple evolutionary origins of prochlorophytes, the chlorophyll $b$-containing prokaryotes. Nature 355, 265-267.

Partensky, F., LaRoche, J., Wyman, K. \& Falkowski, P. G. (1997). The divinyl-chlorophyll $a / b$-protein complexes of two strains of the oxyphototrophic marine prokaryote Prochlorococcus characterization and response to changes in growth irradiance. Photosynth Res 51, 209-222.

Partensky, F., Hess, W. R. \& Vaulot, D. (1999). Prochlorococcus, a marine photosynthetic prokaryote of global significance. Microbiol Mol Biol Rev 63, 106-127.

Penno, S., Campbell, L. \& Hess, W. R. (2000). Presence of phycoerythrin in two strains of Prochlorococcus (cyanobacteria) isolated from the subtropical north Pacific ocean. J Phycol 36, 723-729.

Rocap, G. (2000). Phylogenetic diversity and ecotypic differentiation in the marine cyanobacteria Prochlorococcus and Synechococcus. PhD thesis, Massachusetts Institute of Technology, Cambridge, MA.

Strimmer, K. \& von Haeseler, A. (1996). Quartet puzzling: a quartet maximum likelihood method for reconstructing tree topologies. Mol Biol Evol 13, 964-969.

Swofford, D. L. (1999). PAUP*. Phylogenetic Analysis Using Parsimony ("and Other Methods). Sunderland, MA: Sinauer Associates.

Swofford, D. L., Olsen, G. J., Wadell, P. J. \& Hillis, D. M. (1996). Phylogenetic inference. In Molecular Systematics, pp. 407-415. Edited by D. M. Hillis, C. Moritz \& B. K. Mable. Sunderland, MA: Sinauer Associates.

Tajima, F. (1993). Simple methods for testing molecular clock hypothesis. Genetics 135, 599-607.

Thompson, J. D., Higgins, D. G. \& Gibson, T. J. (1994). CLUSTAL W : improving the sensitivity of progressive multiple sequence alignment through sequence weighting, position-specific gap penalties and weight matrix choice. Nucleic Acids Res 22, 4673-4680.

Ting, C., Rocap, G., King, J. \& Chisholm, S. W. (1999). Characterization of phycoerythrin genes in the chlorophyll $a_{2} / b_{2}-$ containing prokaryote, Prochlorococcus sp. MIT9303. In Photosynthesis: Mechanisms and Effects, vol. I, pp. 225-228. Edited by G. Garab. Dordrecht: Kluwer.

Urbach, E., Robertson, D. L. \& Chisholm, S. W. (1992). Multiple evolutionary origins of prochlorophytes within the cyanobacterial radiation. Nature 355, 267-269.

Urbach, E., Scanlan, D. J., Distel, D. L., Waterbury, J. B. \& Chisholm, S. W. (1998). Rapid diversification of marine picoplankton with dissimilar light harvesting structures inferred from sequences of Prochlorococcus and Synechococcus (cyanobacteria). J Mol Evol 46, 188-201.

Wilbanks, S. M., de Lorimier, R. \& Glazer, A. N. (1991). Phycoerythrins of marine unicellular cyanobacteria. III. Sequence of a class II phycoerythrin. J Biol Chem 266, 9535-9539.

Received 7 December 2000; revised 10 June 2001; accepted 9 July 2001. 